\title{
Análise dos casos de COVID-19 e de dados sociodemográficos nas mesorregiões do estado do Pará
}

Analysis of COVID-19 cases and sociodemographic data in the mesoregions of the state of Pará Análisis de casos COVID-19 y datos sociodemográficos en las mesorregiones del estado del Pará

\author{
Aline Andrade de Sousa \\ ORCID: https://orcid.org/0000-0002-7848-3618 \\ Universidade Federal do Pará, Brasil \\ E-mail: alinesousa@ufpa.br \\ Débora Natassia Carneiro de Pinho \\ ORCID: https://orcid.org/0000-0002-9398-4226 \\ Universidade Federal do Pará, Brasil \\ E-mail: debora.empreend2019@gmail.com \\ Danyela Haykel Claro dos Santos Silva \\ ORCID: https://orcid.org/0000-0002-4836-4116 \\ Universidade Federal do Pará, Brasil \\ E-mail: dany_haykel@hotmail.com \\ Márcia Cristina Freitas da Silva \\ ORCID: https://orcid.org/0000-0002-1315-2084 \\ Universidade Federal do Pará, Brasil \\ E-mail:marciaf@ufpa.br \\ Denis Vieira Gomes Ferreira \\ ORCID: https://orcid.org/0000-0002-2074-7246 \\ Universidade Federal do Pará, Brasil \\ E-mail: denisvgf@ufpa.br \\ Felipe da Costa Soares \\ ORCID: https://orcid.org/0000-0002-6976-3976 \\ Universidade Federal do Pará, Brasil \\ E-mail: felipecsoares4@gmail.com \\ Alexandre Apolo Silva Coelho \\ ORCID: https://orcid.org/0000-0002-0599-5487 \\ Universidade Federal do Pará, Brasil \\ E-mail: alexandre.apolo.coelho@gmail.com \\ Fernando César de Souza Braga \\ ORCID: https://orcid.org/0000-0003-2594-0982 \\ Universidade Federal do Pará, Brasil \\ E-mail: fernando-cesar@outlook.com \\ Antônio Marcos Almeida Bezerra \\ ORCID: https://orcid.org/0000-0002-2585-2125 \\ Universidade Federal do Pará, Brasil \\ E-mail: antonio.bezerra@altamira.ufpa.br \\ Sérgio Beltrão de Andrade Lima \\ ORCID: https://orcid.org/0000-0002-9531-2482 \\ Centro Universitário Metropolitano da Amazônia, Brasil \\ E-mail: sergio.lima@outlook.com \\ Leonardo de Oliveira Rodrigues da Silva \\ ORCID: https://orcid.org/0000-0002-7496-326X \\ Universidade Federal do Pará, Brasil \\ E-mail: leorodrigues@ufpa.br \\ Ademir Ferreira da Silva Júnior \\ ORCID: https://orcid.org/0000-0001-9121-1206 \\ Universidade Federal do Pará, Brasil \\ E-mail: ademirjunior@ufpa.br
}

\section{Resumo}

Introdução: A pandemia do COVID-19, ocasionada pelo SARS-CoV-2, provoca desde infecções assintomáticas até a síndrome respiratória aguda grave, que por vezes, pode ser fatal, necessitando de enfrentamento. Objetivo: Analisar a epidemiologia da COVID-19 nas seis mesorregiões do estado do Pará, correlacionando-a com Índice de Desenvolvimento Humano Médio (IDHm) e o Percentual de Tratamento de esgoto do Estado. Métodos: Trata-se de um estudo descritivo transversal com abordagem quantitativa, realizado através do levantamento de dados secundários dos casos de COVID-19, coletados no período de 07 de março a 30 de maio de 2020, e dados sócio-demográficos das 
seis mesorregiões do estado do Pará. Resultados: Foram notificados 267.700 casos confirmados da COVID-19 em todo o estado do Pará. As mesorregiões do Sudeste paraense e da Região Metropolitana de Belém, apresentaram os maiores valores, com 81.556 e 71.117, respectivamente. Não houve diferença de número de casos entre os gêneros, porém as duas faixas etárias mais acometidas pela infecção estão entre 30 a 39 anos (64.138 casos) e 40 a 49 anos (51.535 casos). Dentre as comorbidades mais prevalentes estão as Cardiopatias e o Diabetes. Conclusão: As maiores taxas de incidência da COVID-19 estão concentradas nas mesorregiões com grande fluxo de pessoas, havendo uma tendência dos aspectos sociodemográficos se relacionarem com a epidemiologia da COVID-19 nas mesorregiões do estado do Pará.

Palavras-chave: COVID-19; Epidemiologia; Mesorregião; Pará.

\begin{abstract}
Introduction: The COVID-19 pandemic, caused by SARS-CoV-2, causes everything from asymptomatic infections to severe acute respiratory syndrome, which can sometimes be fatal, requiring coping. Objective: To analyze the epidemiology of COVID-19 in the six mesoregions of the state of Pará, correlating it with the Average Human Development Index (HDIm) and the percentage of sewage treatment in the state. Methods: This is a cross-sectional descriptive study with a quantitative approach, carried out by collecting secondary data from the cases of COVID-19, collected from March 7 to May 30, 2020, and socio-demographic data from the six mesoregions. of the state of Pará. Results: 267,700 confirmed cases of COVID-19 were reported throughout the state of Pará. The mesoregions of the Southeast of Pará and the Metropolitan Region of Belém, presented the highest values, with 81,556 and 71,117, respectively. There was no difference in the number of cases between genders, however the two age groups most affected by the infection are between 30 and 39 years old (64,138 cases) and 40 to 49 years old (51,535 cases). Among the most prevalent comorbidities are Heart Diseases and Diabetes. Conclusion: The highest incidence rates of COVID-19 are concentrated in mesoregions with a large flow of people, with a tendency for sociodemographic aspects to be related to the epidemiology of COVID-19 in mesoregions of the state of Pará.
\end{abstract}

Keywords: COVID-19; Epidemiology; Mesoregion; Pará.

\title{
Resumen
}

Introducción: La pandemia de COVID-19, causada por el SARS-CoV-2, causa de todo, desde infecciones asintomáticas hasta síndrome respiratorio agudo severo, que a veces puede ser fatal y requiere afrontamiento. Objetivo: Analizar la epidemiología del COVID-19 en las seis mesorregiones del estado de Pará, correlacionándolo con el Índice de Desarrollo Humano Promedio (IDH) y el porcentaje de tratamiento de aguas residuales en el estado. Métodos: Se trata de un estudio descriptivo transversal con enfoque cuantitativo, realizado mediante la recolección de datos secundarios de los casos de COVID-19, recolectados del 7 de marzo al 30 de mayo de 2020, y datos sociodemográficos de las seis mesorregiones. De el estado de Pará. Resultados: se notificaron 267.700 casos confirmados de COVID-19 en todo el estado de Pará, siendo las mesorregiones del Sureste de Pará y la Región Metropolitana de Belém las que presentaron los valores más altos, con 81.556 y 71.117, respectivamente. No hubo diferencia en el número de casos entre sexos, sin embargo, los dos grupos de edad más afectados por la infección son entre 30 y 39 años (64,138 casos) y 40 a 49 años (51,535 casos). Entre las comorbilidades más prevalentes se encuentran las enfermedades cardíacas y la diabetes. Conclusión: Las mayores tasas de incidencia de COVID-19 se concentran en mesorregiones con gran flujo de personas, con tendencia a que los aspectos sociodemográficos estén relacionados con la epidemiología de COVID-19 en mesorregiones del estado de Pará.

Palabras clave: COVID-19; Epidemiología; Mesorregión; Pará.

\section{Introdução}

Casos de pneumonia de origem desconhecida, em dezembro de 2019, em Wuhan, província de Hubei-China alertaram sobre uma nova doença respiratória denominada COVID-19, ocasionada pelo novo coronavírus SARS-CoV-2, que a partir de então, iniciou sua disseminação pelo mundo. Em 11 de março de 2020 foi considerada emergência de saúde pública, de importância internacional e classificada como Pandemia pela Organização Mundial da Saúde - OMS (Zhu et al., 2020). A OMS instituiu as medidas essenciais para a prevenção e enfrentamento a serem adotadas, dentre as quais estão incluídas a higienização das mãos com água e sabão sempre que possível e uso de álcool em gel, em situações em que o acesso à água e ao sabão não fosse possível (Chan et al., 2020).

Historicamente, a humanidade experimentou outras pandemias, algumas com ciclos repetidos por séculos, como a varíola e o sarampo, ou por décadas, como as de cólera. Ainda podem ser citadas as pandemias de gripe por H1N1 em 1918, por H2N2 em 1957-58, por H3N3 em 1968-69 e por H5N1 nos anos 2000, conhecidas, respectivamente, como "gripe espanhola”, “gripe asiática”, “gripe de Hong-Kong” e "gripe aviária” (Souza et al., 2020; Bevenutu et al., 2020). Uma revisão 
sistemática global avaliou o perfil epidemiológico da COVID-19 e verificou que a taxa de mortalidade por essa causa chega a 0,3\% da população (Maciel et al, 2020).

Dentre as ocorrências recentes de doenças de potencial pandêmico, dos Séculos XX e XXI, o SARS-CoV-2 é a terceira ameaça global à saúde pública (Nassiri et al., 2020). A pandemia da COVID-19 apresentou-se como um dos maiores desafios sanitários em escala global deste século. A falta de conhecimento científico sobre o novo coronavírus, sua alta velocidade de disseminação e capacidade de provocar mortes em populações vulneráveis, geram incertezas sobre quais seriam as melhores estratégias a serem utilizadas para o enfrentamento da epidemia em diferentes partes do mundo (Bevenutu et al., 2020).

Trata-se de uma doença causada por um vírus zoonótico que apresenta um quadro clínico que vai desde infecções assintomáticas até uma síndrome respiratória aguda grave (SRAG) e por vezes, à morte (Wang et al., 2020). Dentre os que necessitam de atendimento hospitalar (cerca de 20\%), podem necessitar de suporte para tratamento respiratório em 5\% dos casos (OMS, 2020). Indivíduos de qualquer faixa etária podem apresentar SRAG pelo coronavírus, embora seja mais comum em adultos de meia idade e idosos. A idade é um fator de risco importante para a doença grave, complicações e óbito (Dias et al, 2020).

Além da idade, as condições precárias de habitação e saneamento, juntamente com a alta prevalência de doenças crônicas, como hipertensão e diabetes, que são fatores de risco para a COVID-19, são determinantes para o registro de elevadas taxas de contaminação e de mortalidade, colocando o Brasil entre os mais atingidos pela doença (Souza et al., 2020).

No Brasil, a doença tem se apresentado de forma desfavorável, com alarmantes taxas de contaminação e de mortalidade, colocando o país entre os mais atingidos. O registro de aumento agudo de detecção frente aos demais tipos de doenças causadas por vírus respiratórios monitorados demonstra que a COVID-19 possui comportamento incomum e representa elevado risco para o Sistema Único de Saúde - SUS (Silva et al, 2020). Em todas as regiões do país, populações têm sido acometidas, repercutindo impactos sociais, epidemiológicos, sanitários, econômicos e políticos, sem precedentes na história recente das epidemias (Souza et al., 2020; Bevenutu et al., 2020; Oliveira et al., 2020).

Nessa perspectiva, o Brasil, na semana epidemiológica (SE) 21 (relativa a 23 de maio), ocupou a segunda colocação mundial em casos confirmados e sexto lugar em número de casos. A primeira unidade federativa a apresentar caso confirmado foi São Paulo ( $\left.9^{\mathrm{a}} \mathrm{SE}\right)$. No Pará, o primeiro diagnóstico ocorreu na $12^{\mathrm{a}} \mathrm{SE}$ e o primeiro óbito na $14^{\mathrm{a}} \mathrm{SE}$. A utilização das SE se faz necessária para a identificação da fase da pandemia na qual a unidade federativa se encontra, tornando viáveis as comparações com outras regiões do país (Ministério da Saúde - MS, 2020).

Por se tratar de uma doença e de uma situação nova, as lacunas de informação e conhecimento ainda são muito grandes: taxas de letalidade, potencial de transmissão, tratamento, existência de outros efeitos ou sequelas no organismo dos que foram infectados, todas essas informações ainda são preliminares (Bevenutu et al., 2020), o que pode apresentar um quadro bem pior no Estado do Pará. Nesse contexto, informações de qualidade baseadas em conhecimento científico, além de ações de educação em saúde são necessários para o enfrentamento da COVID-19 dentro dos municípios que compõem o estado do Pará.

O Pará é constituído por 6 mesorregiões: Baixo Amazonas, Marajó, Metropolitana de Belém, Nordeste, Sudeste e Sudoeste (Figura 1). Sendo a Região Metropolitana de Belém formada por 11 municípios, o Nordeste Paraense possui 49 municípios, a mesorregião do Marajó possui 16 municípios, o Baixo Amazonas 15 municípios, o Sudoeste também 14 municípios e o Sudeste Paraense é formado por 39 municípios (Instituto Brasileiro de Geografia e Estatística - IBGE, 2020). 
Figura 1. Mesorregiões do Estado do Pará.

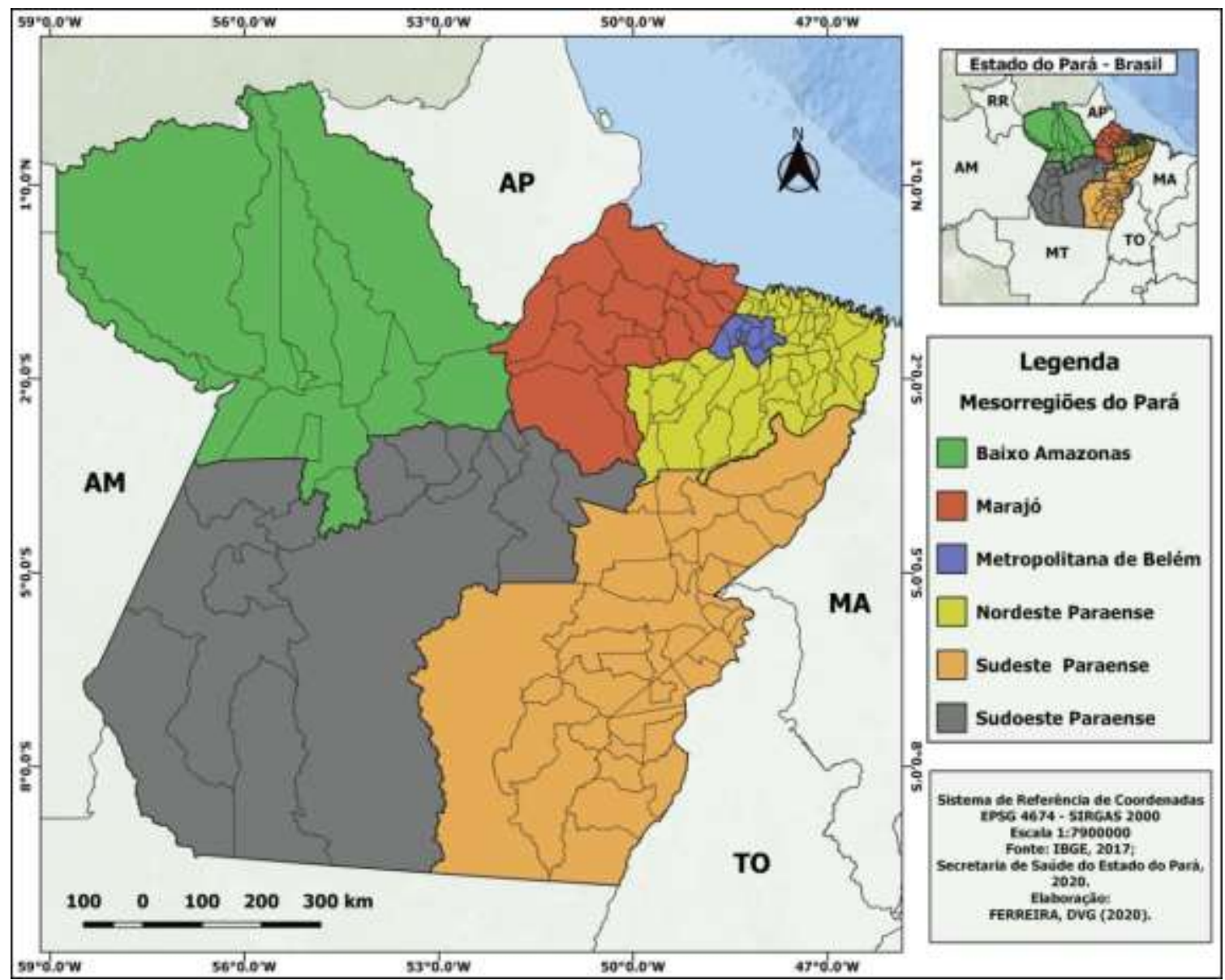

Fonte: IBGE, elaboração Ferreira (2020).

Dentre a maioria dos municípios das mesorregiões que compõem o Estado do Pará, o saneamento básico é avaliado como precário, abaixo do percentual nacional (IBGE, 2020). De acordo com o censo (IBGE, 2010), a desigualdade social entre ricos e pobres impacta diretamente na qualidade de vida desses grupos sociais, desde a alimentação até o saneamento básico. Segundo o mesmo censo, $45 \%$ da população brasileira não tem acesso ao tratamento de esgoto. No Brasil estima-se que aproximadamente 13 milhões de pessoas estejam vivendo sem acesso ao saneamento básico e cerca de 7\% não possui banheiro apropriado em suas residências, demonstrando que a maioria da população não conta com um serviço de garantia mínima para a preservação das condições básicas de saúde e prevenção de inúmeras doenças (Fundo das Nações Unidas para a Infância UNICEF, 2011).

Nesse contexto, a emergência da COVID-19 como pandemia, ocasionou uma série de especulações relacionadas aos fatores que poderiam ter influência sobre a disseminação do vírus, além das já comprovadas (Cascella et al., 2020; Doremalen et al., 2020; OMS/WHO, 2020), voltando-se também, às questões relacionadas à aspectos sociodemográficos da população, pois a urbanização, a ampliação da cobertura do saneamento básico, a melhoria das condições habitacionais e a introdução de novas tecnologias de saúde, bem como o acesso aos serviços de saúde na atenção primária e a outros determinantes sociais, reduziram a mortalidade por doenças infecciosas no Brasil, durante o século XX (Luna \& Silva, 2013). Embora o trabalho de Luna e Silva (2013) refira-se ao século XX, podemos extrapolar essa configuração ao século XXI, como demonstrado por Doremalen e colaboradores (2020).

A introdução ou reintrodução de doenças infecciosas no mundo, tem trazido preocupação nas agendas dos governos onde a saúde pública é a prioridade e atividades de controle dessas doenças precisam de atenção urgente. Nesse contexto é de 
extrema necessidade que se fortaleça a cobertura do saneamento básico e vacinação, da rede de serviços de saúde e do sistema de vigilância (Waldman \& Sato, 2016). Além disso, boas condições de vida e infraestrutura alcançadas em centros urbanos que apresentam altos Índices de Desenvolvimento Humano, possibilitam melhores condições na atenção à saúde da população (Kerr-Pontes et al. 2006, Ledeberg, 1997; Beaglehole \& Bonita, 1997).

Nesse contexto, a OMS, entendendo a diversidade sanitária mundial, define através do decreto de Emergência de Saúde Pública de Âmbito Internacional que medidas sanitárias protetivas contra a disseminação sejam tomadas. Isso referenda o fechamento de fronteiras, o aumento no critério de fiscalização em portos e aeroportos, permite a flexibilização na importação de equipamentos de uso em ambientes de prestação de serviços de saúde, tanto quanto a adoção de rotinas diferenciadas de trabalho, como medidas de monitoramento de outros parâmetros de saúde de imigrantes, trabalhadores ou turistas, como aferição de temperatura ou realização de exames. Todas essas decisões cabem aos governos de cada país, estado ou município, obedecendo o ordenamento jurídico de cada nação (OMS/WHO, 2005).

Assim como preconizado pela OMS, algumas medidas foram adotadas desde o aparecimento dos primeiros casos de COVID-19 nos municípios do Estado do Pará, como suspensão das aulas, fechamento do comércio, bares e restaurantes onde somente serviços essenciais como supermercados e farmácias, além de hospitais e estabelecimentos de saúde, puderam continuar funcionando, mas em horários reduzidos (Decreto $\mathrm{n}^{\circ} 1.195$, de 20 de março de 2020), com o intuito de reduzir o contato social e consequentemente a disseminação do vírus, reduzindo e desacelerando a curva de contaminação.

Porém, mesmo com a adoção dessas medidas, a elevada infectividade do agente etiológico da COVID-19 e a ausência de imunidade prévia na população humana faz com que o crescimento do número de casos seja exponencial e cause enorme impacto no sistema de saúde. Dada a rápida disseminação do vírus, estudos epidemiológicos são determinantes no acompanhamento de situações como a Pandemia da COVID-19, neste contexto, o presente estudo tem o intuito de apresentar uma avaliação do perfil epidemiológico dos casos de COVID-19 nas mesorregiões do estado do Pará correlacionando-os ao Índice de Desenvolvimento Humano (IDH) médio e ao Tratamento de esgoto do Estado do Pará.

\section{Metodologia}

Este é um estudo do tipo descritivo transversal com abordagem quantitativa, realizado através do levantamento de dados secundários referentes aos casos de COVID-19 nas mesorregiões do estado do Pará e baseados na metodologia de Pereira et. al (2018).

Foi utilizado a técnica de documentos como instrumento de coleta de dados. Os documentos analisados foram registros estatísticos provenientes do Sistema de Vigilância da Secretaria de Saúde Pública do Estado do Pará (SESPA) e do Instituto Brasileiro de Geografia e Estatística (IBGE).

Os dados coletados referentes ao número de casos de COVID-19 no estado do Pará foram coletados no dia 30 de maio de 2020 às 19:30 horas no site da SESPA e fazem referência ao período de 07 de março (registro do primeiro caso no Pará) a 30 de maio de 2020. Já os dados sócio demográficos, referentes ao último censo do IBGE das seis mesorregiões (Baixo Amazonas, Marajó, Metropolitana de Belém, Nordeste Paraense, Sudoeste Paraense e Sudeste Paraense) que compõem o estado do Pará, foram coletadas dia 12 de setembro às 12:00 horas. Posteriormente, com esses dados, foi calculado a média aritmética de cada mesorregião.

Todos os casos confirmados de infecção humana pelo novo Coronavírus foram definidos pela confirmação laboratorial por um ou mais dos testes RT-PCR, específicos para o vírus, e descartados os casos não confirmados ou apenas suspeitos.

A coleta de dados abrangeu variáveis diversas, dependendo da fonte, como apresentado no quadro a seguir (Quadro $1)$ 
Quadro 1 - Variáveis coletadas nas fontes de dados SESPA e IBGE.

\begin{tabular}{|c|c|}
\hline Variáveis -SESPA & Variáveis - IBGE \\
\hline $\mathrm{N}^{\circ}$ Casos confirmados & Índice de Desenvolvimento Humano (IDH) médio \\
\hline $\mathrm{N}^{\circ}$ Casos Recuperados & Tratamento de esgoto (\%) \\
\hline $\mathrm{N}^{\mathbf{o}}$ de Óbitos & Densidade demográfica $\left(\mathrm{hab}^{\mathrm{k}} / \mathrm{km}^{2}\right)$ \\
\hline Distribuição etária de casos confirmados & \\
\hline Sexo dos casos confirmados \\
\hline Comorbidades prevalentes \\
\hline Letalidade
\end{tabular}

Fonte: Autores.

Os dados coletados foram tabulados com o auxílio do programa de planilhas eletrônicas MS Office Excel ® e analisados por meio do software BIOSTAT, versão 5.0. Os resultados foram apresentados segundo distribuições de frequências e medidas estatísticas descritivas tais como: média aritmética, desvio-padrão, para as variáveis quantitativas, admitindo um $\mathrm{p}<0,001$.

Por se tratar de estudo realizado com dados secundários, acessado de um banco de domínio público, não foi necessário submeter o projeto ao Comitê de Ética em Pesquisa com Seres Humanos.

\section{Resultados}

Foram notificados, no Sistema de Vigilância Epidemiológica da SESPA, de 07 de março a 30 de maio de 2020 , 267.700 casos confirmados da COVID-19 em todo o estado do Pará, obtendo diferente distribuição dos casos em cada mesorregião, como demonstrado no Figura 2. As duas mesorregiões que apresentaram maior número de casos confirmados para COVID-19 (acima da média do estado) foram o Sudeste paraense e a mesorregião Metropolitana de Belém, com 81.556 e 71.117, respectivamente, enquanto os menores números foram encontrados no Sudoeste paraense (24.384) e Marajó (14.054), como indicado no Figura 2. 
Figura 2. Casos confirmados e notificados da COVID-19 por mesorregião do estado do Pará de 07 de março a 30 de maio de 2020.

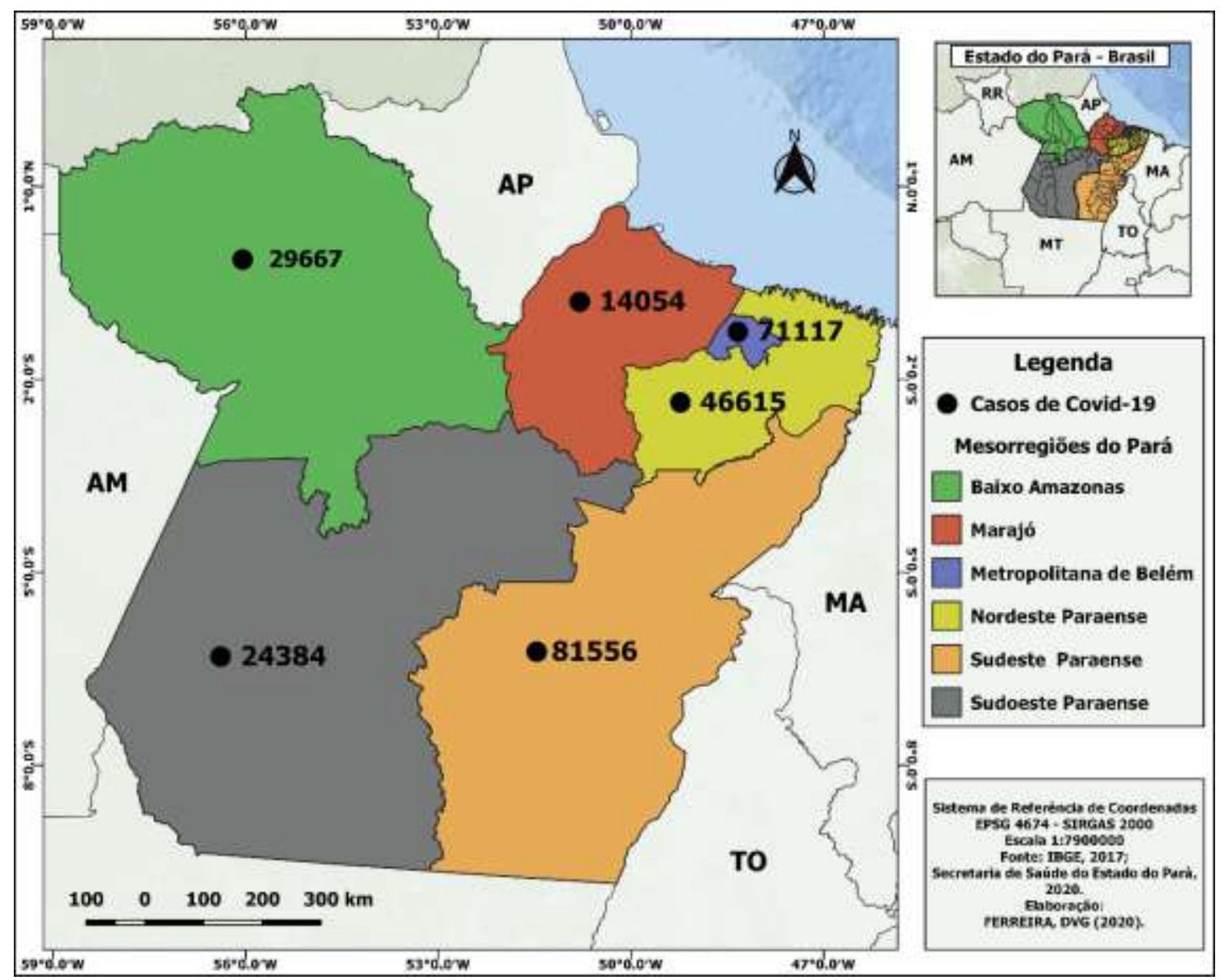

Fonte: IBGE e SESPA, elaboração Ferreira (2020).

Dentre os casos confirmados de COVID-19 no estado do Pará houve uma distribuição linear entre os sexos, não havendo diferença estatística entre os gêneros (Figura 3).

Figura 3. Distribuição dos casos confirmados da COVID-19 por gênero por mesorregião do estado do Pará de 07 de março a 30 de maio de 2020.

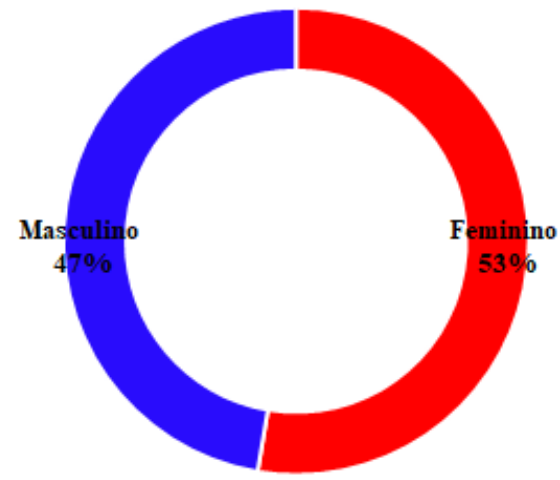

Fonte: Autores, a partir de dados da Vigilância Epidemiológica - SESPA. 
As duas faixas etárias mais acometidas pela infecção estão entre 30 a 39 anos (64.138 casos) e 40 a 49 anos (51.535 casos), em contrapartida, as duas com menor taxa de infecção foram entre 0 a 9 anos e 80 anos ou mais (Figura 4).

Figura 4. Perfil de distribuição dos casos confirmados da COVID-19 para cada faixa etária no estado do Pará de 07 de março a 30 de maio de 2020 .

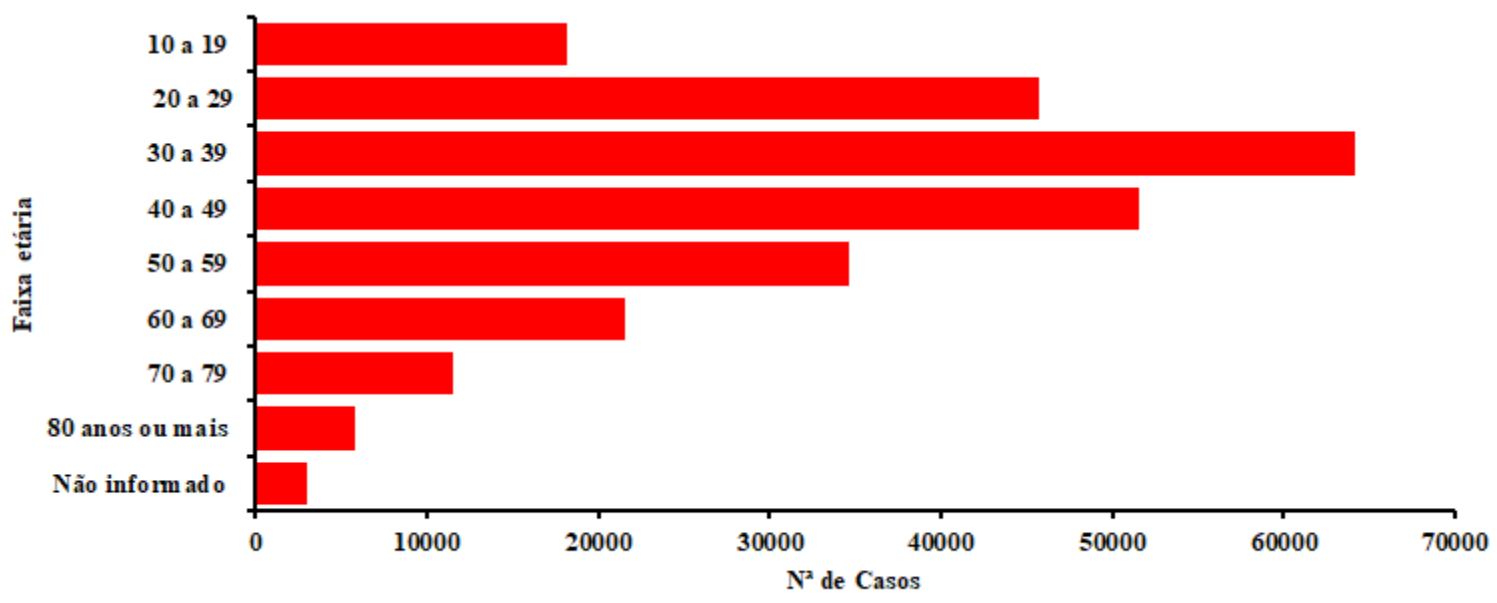

Fonte: Autores, a partir de dados da Vigilância Epidemiológica - SESPA.

Em relação às comorbidades mais prevalentes entre os casos confirmados da COVID-19, as Cardiopatias e o Diabetes foram as mais frequentes, com frequências de 4,24\% e 3,24\%, respectivamente (Figura 5).

Figura 5. Comorbidades mais frequentes entre os casos confirmados da Covid-19 (SARS-CoV-2) no Pará de 07 de março a 30 de maio de 2020 .

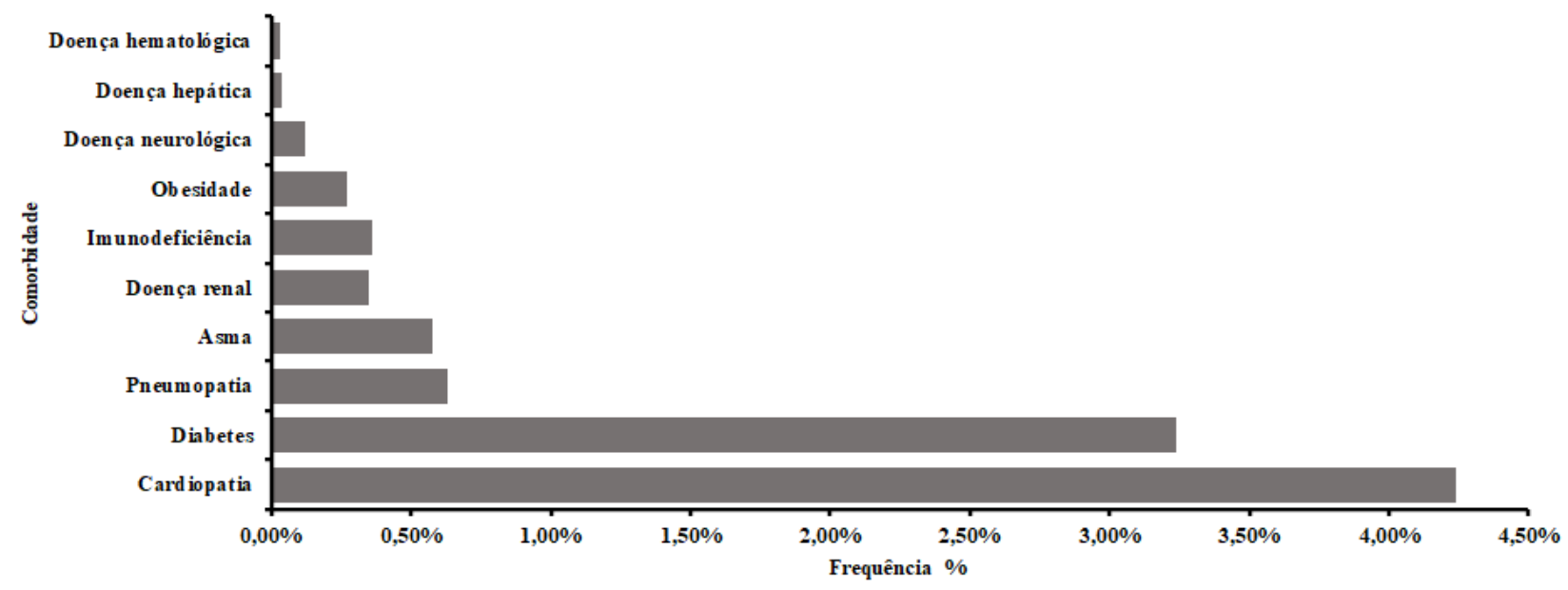

Fonte: Autores, a partir de dados da Vigilância Epidemiológica - SESPA.

Relacionando as variáveis taxa de incidência de casos confirmados (TI), Índice de Desenvolvimento Humano médio (IDHm) e Percentual de tratamento de esgoto (\% TE) no estado do Pará foi observado que as três mesorregiões com maior incidência de COVID-19 foram Sudeste, Nordeste e Metropolitana de Belém, de forma que duas (Nordeste paraense e Sudeste paraense) delas também possuem IDHm baixo (0,567 e 0,604, respectivamente) e uma delas baixo \% TE (Sudeste, com 
12,71\%). Embora a mesorregião do Marajó esteja entre as três mesorregiões com os piores IDHm e \%TE, não está incluída entre as três mesorregiões com maior incidência da COVID-19 (**) (Figura 6 e Quadro 2).

A partir da relação entre TI, do Índice de Desenvolvimento Humano médio (IDHm) e do Percentual de tratamento de esgoto (\%TE) no estado do Pará observamos que a mesorregião Sudeste Paraense apresentou o maior Taxa de Incidência $(93,84)$, possuindo IDHm de $(0,604)$, que está abaixo do estadual $(0,585)$ (dado não mostrado na tabela), além de possuir uma das piores Taxas de Tratamento de esgoto do estado com a média de (12,71\%), bem abaixo da média estadual de 15,02\% (dado não mostrado na tabela).

A terceira região com o maior índice de infecção é a região Nordeste Paraense com $(53,64)$, que possui IDHm de $(0,567)$, também abaixo da média do estado, a qual a (\%TE) é de $(16,56 \%)$ dos esgotos tratados. A região Metropolitana de Belém tem o maior IDHm do Estado do Pará $(0,653)$, bem como o maior \%TE $(25,90 \%)$, contudo apresenta a segunda maior Taxa de Incidência $(81,83)$ (Quadro 2).

Analisando a relação entre a densidade populacional (habitante $/ \mathrm{km} 2$ ), a incidência e o $\mathrm{n}^{\circ}$ de casos confirmados para COVID-19 durante o período do estudo, observou-se que há uma tendência importante, que mostra que as mesorregiões com maior densidade populacional também são as que apresentam maior taxa de incidência e $\mathrm{n}^{\circ}$ de casos confirmados para a COVID-19 (\#), como ocorre nas mesorregiões Metropolitana de Belém, Nordeste Paraense e Sudeste do Pará (Quadro 2).

Quadro 2. Quadro mostrando a densidade populacional, o $\mathrm{n}^{\mathrm{o}}$ de casos confirmados, a taxa de incidência dos casos confirmados para COVID-19, o Índice de Desenvolvimento Humano (IDH) médio e a Porcentagem de tratamento de esgoto, por mesorregião paraense registrados de 07 de março a 30 de maio de 2020. Taxa de incidência $=n^{\circ}$ de casos confirmados/população 8.690 .745 X 10.000 .

\begin{tabular}{|l|c|c|c|c|c|}
\hline \multicolumn{1}{|c|}{ Mesorregião } & $\begin{array}{c}\text { Densidade } \\
\text { demográfica } \\
\left(\mathbf{h a b} / \mathbf{k m}^{2}\right)\end{array}$ & $\begin{array}{c}\mathbf{N}^{\mathbf{0}} \text { casos } \\
\text { confirmad } \\
\text { os } \\
\text { COVID-19 }\end{array}$ & $\begin{array}{c}\text { Taxa de } \\
\text { incidência } \\
\text { de casos } \\
\text { confirmados } \\
\text { (TI) }\end{array}$ & $\begin{array}{c}\text { IDH médio } \\
- \text { (IDHm) }\end{array}$ & $\begin{array}{c}\text { Tratamento } \\
\text { de esgoto } \\
\text { (\% média) - } \\
(\% \text { TE) }\end{array}$ \\
\hline Baixo Amazonas & 2,4 & 29667 & 34,14 & 0,588 & 13,98 \\
\hline Marajó & 5,5 & 14054 & 16,17 & $\mathbf{0 , 5 1 9}$ & $\mathbf{1 1 , 7 1}$ \\
\hline Metropolitana de Belém\# & $\mathbf{3 9 3 , 4}$ & $\mathbf{7 1 1 1 7}$ & $\mathbf{8 1 , 8 3}$ & 0,653 & 25,90 \\
\hline Nordeste paraense\# & $\mathbf{2 4 , 4}$ & $\mathbf{4 6 6 1 5}$ & $\mathbf{5 3 , 6 4}$ & $\mathbf{0 , 5 6 7}$ & 16,56 \\
\hline Sudoeste paraense* & 1,3 & 24384 & 28,06 & $\mathbf{0 , 5 7 8}$ & $\mathbf{9 , 2 3}$ \\
\hline Sudeste paraense**\# & $\mathbf{6 , 6}$ & $\mathbf{8 1 5 5 6}$ & $\mathbf{9 3 , 8 4}$ & 0,604 & $\mathbf{1 2 , 7 1}$ \\
\hline
\end{tabular}

Fonte: Autores, a partir de dados da Vigilância Epidemiológica - SESPA e IBGE. Menor \%TE (*); maior incidência da COVID-19 (**) e \# tendência de relação entre a densidade populacional (habitante/km2), a incidência e o $\mathrm{n}^{\circ}$ de casos confirmados para COVID-19.

As três mesorregiões que apresentaram maior percentual de letalidade da infecção pela COVID-19 foram a Metropolitana de Belém, Marajó e Sudeste paraense, com 10\%, 7,38\% e 5,62\%, respectivamente (Figura 6). 
Figura 6. Letalidade para COVID-19, por mesorregião paraense Pará de 07 de março a 30 de maio de 2020.

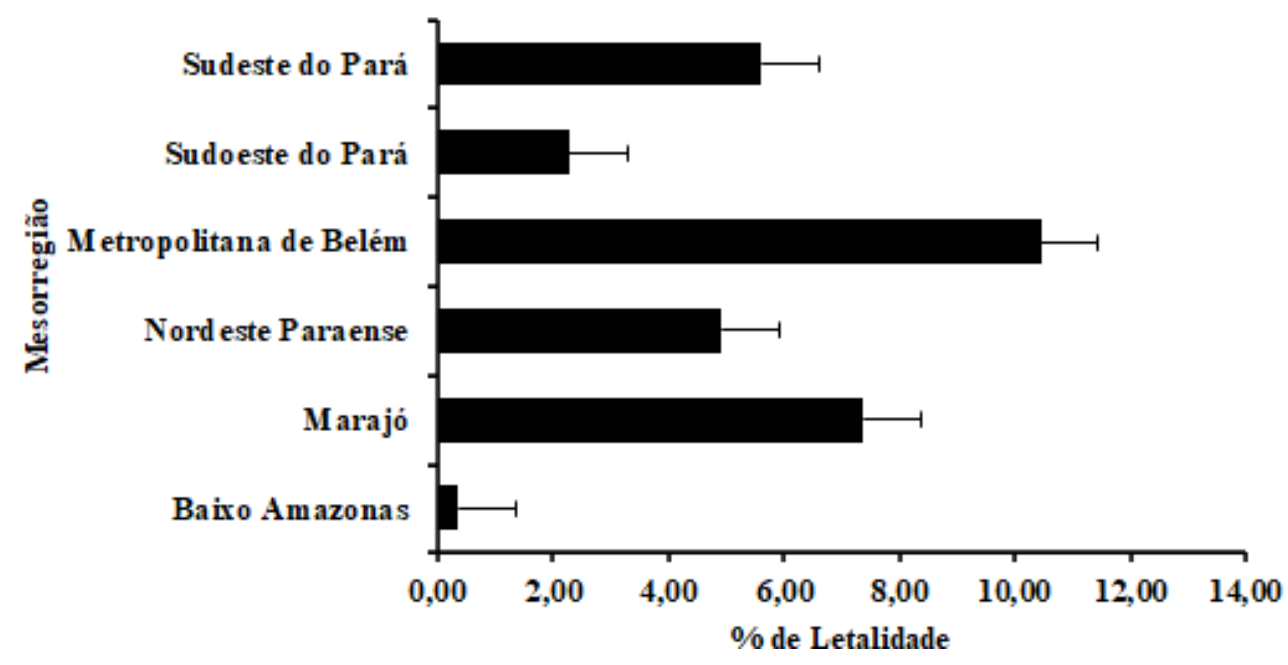

Fonte: Autores, a partir de dados da Vigilância Epidemiológica - SESPA e IBGE.

Os dados totais do estado do Pará, para o número de casos recuperados e óbitos, indicaram que a maioria dos casos confirmados da COVID-19 se recupera da doença (93,37\%), enquanto a taxa percentual de óbitos chega a 2,57\%, (Figura 7).

Figura 7. Relação entre as taxas de casos recuperados e óbitos por Covid-19, por mesorregião paraense de 07 de março a 30 de maio de 2020 .

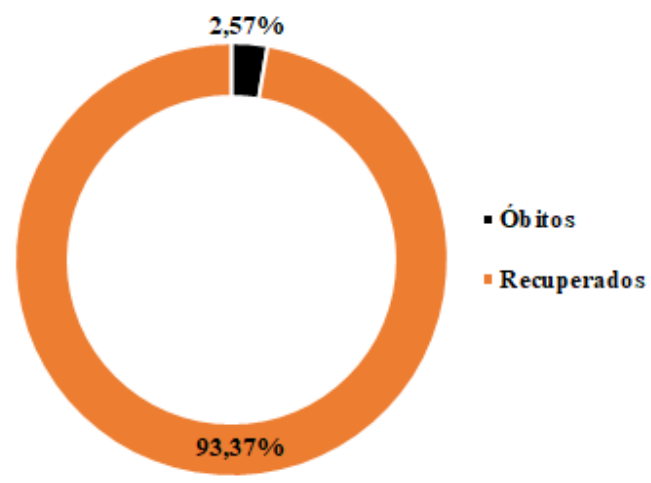

Fonte: Autores, a partir de dados da Vigilância Epidemiológica - SESPA.

\section{Discussão}

A COVID-19 é uma doença respiratória aguda grave, causada pelo novo coronavírus (SARS-CoV-2), que surgiu no final de 2019 na China, se espalhando rapidamente por todos os continentes do mundo ainda no início de 2020, demonstrando sua alta transmissibilidade e letalidade, ocasionando sérios problemas à população (Silva et al., 2020; Muralidar et al., 2020). O registro de maior detecção frente aos demais tipos de vírus respiratórios monitorados mostra que a COVID-19 possui comportamento incomum e representa elevado risco para o Sistema Único de Saúde - SUS (Silva et al, 2020).

No mundo, desde a descoberta da doença até o dia 17 de dezembro de 2020, foram registrados 72.851 .747 casos confirmados de COVID-19, 19.148.430 somente nas Américas e no Brasil, aproximadamente 6.388.526 casos (OMS/WHO, 2020), com mais de 1.400.000 óbitos (Silva et al, 2020), ressaltando ainda mais sua alta transmissibilidade. No Pará, no período de 07 de Março a 30 de Maio de 2020, foram notificados no Sistema de Vigilância Epidemiológica da SESPA, 267.700 casos confirmados da COVID-19. 
O estado do Pará apresenta 144 municípios divididos em seis mesorregiões com pluralidade de características ambientais e socioeconômicas. As mesorregiões mais impactadas com grande número de casos confirmados de COVID-19 foram o Sudeste paraense e a mesorregião Metropolitana de Belém. O que pode, talvez ser explicado, pelo grande dinamismo socioeconômico e pelas profundas transformações na base socioprodutiva dessa região (Monteiro Neto et al., 2017), ou pode estar relacionado ao grande fluxo de pessoas, a serviço e/ou turisticamente, e por sediar a capital paraense, enquanto os menores números de casos confirmados foram encontrados no Sudoeste paraense e no Marajó, o que talvez possa ser justificado por estarem entre as mesorregiões com as menores densidades populacionais do Estado do Pará (Silva et al., 2020).

Embora o trabalho realizado por Sousa (2020) em comunidades tradicionais corrobore os resultados do presente artigo, outros trabalhos apontam o sexo masculino como o mais infectado (CHEN et al, 2020), talvez pela ação desenvolvida pelos cromossomos $\mathrm{X}$ da mulher e pela atuação dos hormônios sexuais feminimos no sistema imunológico do corpo (Jaillon et al., 2019; Lima et al., 2020).

A faixa etária acima de 40 anos é apontada, em alguns estudos, como a mais comprometida durante a pandemia da COVID-19, porém alguns fatores influenciam diretamente nesta classificação, destacando as comorbidades associadas à idade, enquadrando o indivíduo no grupo de risco (Lima et al., 2020; Chen et al., 2020). As duas faixas etárias mais comprometidas neste trabalho foram entre 30-39 e 40-49 anos, corroborando os estudos de Silva et al. (2020), Lima et al. (2020), Chen et al. (2020) e Sousa et al. (2020), este último realizado em comunidades indígenas. Acredita-se que as faixas etárias mais acometidas fazem parte das equipes de serviços essenciais que trabalham na linha de frente no enfrentamento da COVID-19 ou trabalhadores do mercado informal que não puderam cumprir a quarentena e o isolamento físico. Neste trabalho foi demonstrado que as faixas etárias menos acometidas correspondem às crianças abaixo de 9 anos e idosos acima de 80 anos, assim como observado por Silva e colaboradores (2020).

No Brasil vivem mais de 28 milhões de pessoas idosas, na faixa etária igual ou superior a 60 anos, representando cerca de 13\% da população nacional. Segundo a Projeção da População divulgada pelo IBGE em 2018, essa porcentagem irá dobrar nas próximas décadas (https://agenciadenoticias.ibge.gov.br/). O trabalho realizado por Muralidar et al. (2020) aponta que a população idosa representa o grupo mais acometido pela forma grave da COVID-19, porém nossos resultados evidenciam que as faixas etárias a partir de 60-69 anos e abaixo dos 19 anos, apresentam menor número de casos confirmados, porém não temos informações acerca da gravidade desses casos.

Analisando as comorbidades mais prevalentes entre os casos confirmados da COVID-19 nas mesorregiões do Pará, as Cardiopatias e o Diabetes foram as mais frequentes, e de acordo com Pachiega et al. (2020) e Silva et al. (2020) também foram as comorbidades mais prevalentes no Brasil. Com base no estudo realizado por Muralidar et al. (2020), pessoas com comorbidades anteriores são altamente suscetíveis aos efeitos graves da COVID-19, o que contribui ainda para a regulação positiva de citocinas e para o desenvolvimento da Síndrome da Angústia Respiratória Aguda (SARA).

A disseminação da doença ocorreu em curto espaço de tempo, deixando de ser um evento local para uma pandemia global em menos de 04 meses. Países como Estados Unidos, Itália e Espanha, registraram um aumento exponencial dos casos, repercutindo no comprometimento das estruturas e serviços de saúde locais. No período de coleta dos dados, o número maior de casos estava nas capitais e municípios próximos a elas (MS, 2020; Silva et al., 2020), porém em nosso trabalho, a mesorregião Metropolitana de Belém apresentou a $2^{\circ}$ maior taxa de incidência (TI), acompanhada do Sudeste, na $1^{\mathrm{a}}$ colocação.

A região Metropolitana de Belém e o Nordeste Paraense, apesar de apresentarem os melhores índices percentuais de Tratamento de esgoto (\% TE) e do pequeno avanço em seu desenvolvimento estrutural, ainda demonstram baixa distribuição de rendimentos entre a população (IBGE, 2010; Luna \& Silva, 2013; Silva et al., 2020). Nestas mesorregiões, há municípios com reduzida arborização, lixo acumulado, esgoto a céu aberto e condições precárias de moradia, assim como as outras capitais da região Norte apresenta condições ruins de bem estar urbano (IBGE, 2010; Andrade et al., 2019). 
Contudo, a aglomeração e ausência de acesso constante à água e esgoto tratados são indicadores socioeconômicos desfavoráveis que refletem a qualidade de vida da maioria da população, ampliando os impactos e dificultam as estratégias de enfrentamento da COVID-19 (Mascarenhas et al, 2020).

O Pará é classificado como médio na faixa de desenvolvimento humano (0,600 - 0,699). O Nordeste paraense e o Marajó apresentam os menores IDHm do Estado (0,567 e 0,519), respectivamente, apresentando baixo acesso a bens e serviços, além de baixos índices de bem estar urbano e condições ambientais, ao redor das residências, e habitacionais ruins, colocando os municípios que as constituem, na lista das piores condições urbanas do Brasil (Andrade et al., 2019). Além do IDH, também há uma relação entre a densidade populacional e a taxa de incidência de casos confirmados de COVID-19 (Silva et al., 2020), corroborando os resultados de nosso estudo.

A correlação entre a incidência de COVID-19 e os indicadores sociais, sociodemográficos e de saúde, infere que as capitais com maior desenvolvimento econômico, populacional e de saúde foram as mais acometidas pelo coronavírus no Brasil. Tal constatação deve-se, principalmente, pelo maior fluxo de viajantes. Diante disso, medidas restritivas de mobilidade populacional, assim como de afastamento social, podem ser mais efetivas se direcionadas para as capitais com grande fluxo de pessoas (Silva et al., 2020)

De acordo com o MS (2020), a taxa de letalidade da COVID-19 no país, até o mês de novembro foi de 2,7\%, enquanto que a maior taxa de letalidade encontrada para o estado do Pará, no mesmo período, nas mesorregiões Metropolitana de Belém, foi de $10 \%$, quase 4 vezes maior que a taxa nacional, e Marajó com 7,38\%, o que pode talvez ser explicado, pela precariedade do sistema de saúde (Silva et al, 2020), pela dificuldade de acesso em vários municípios paraenses (Sousa et al., 2020) e pelos menores índices de indicadores sociais do Brasil, no caso da mesorregião do Marajó (Andrade et al., 2019).

Comparando as taxas de recuperação e de óbitos no Brasil e no estado do Pará, no período de março a novembro de 2020, temos que a porcentagem nacional de recuperados é de 88,33\% (Brasil, 2020), enquanto que a taxa estadual correspondeu a 93,37\% dos casos, 5,04\% acima da média do país, o que pode ser resultado da adoção de medidas restritivas mais severas, como o Lockdown adotado no Pará através do Decreto no 1.195, de 20 de março de 2020.

O maior número de óbitos (3.198) ocorreu na mesorregião Metropolitana de Belém, o que pode estar relacionado à superlotação do sistema de saúde, às comorbidades associadas e à espera pelo uso de unidade de tratamento intensivo, que são fatores que podem aumentar o risco de desenvolver a forma grave do COVID-19 (Chen et al., 2020; Wang et al., 2020). Entretanto somente 2,57\% da população contaminada com o vírus foi a óbito, dos 267.700 casos confirmados de COVID-19, 260.820 pessoas recuperaram-se da doença durante o período analisado. Até o momento da coleta dos dados apresentados neste estudo, apenas duas cidades no estado do Pará (Faro, na mesorregião Baixo Amazonas e Água Azul do Norte, no Sudeste Paraense) não apresentaram óbitos confirmados de COVID-19.

\section{Conclusão}

Devido ao rápido aumento do número de casos do novo coronavírus em todos os estados do Brasil, ações de monitoramento e estudos de perfis epidemiológicos são fundamentais para conhecer a evolução da pandemia, auxiliando com estratégias adequadas para o enfrentamento da doença.

O presente estudo descreveu o perfil epidemiológico da COVID-19 e sua relação com os dados sócio demográficos disponibilizados pelo IBGE nas mesorregiões do estado do Pará. Nossos resultados demonstraram que as maiores taxas de incidência da COVID-19 estão concentradas nas mesorregiões com grande fluxo de pessoas, e que há uma tendência dos aspectos sociodemográficos de se relacionarem com a epidemiologia da COVID-19 nas mesorregiões do estado do Pará, porém mais estudos devem ser realizados, com o intuito de compreender essa relação. 
As informações utilizadas para a elaboração da pesquisa foram obtidas nas bases de dados oficiais do Sistema de Vigilância da Secretaria de Saúde Pública do estado do Pará (SESPA), o que depende diretamente da atualização das informações pelas unidades de saúde municipais e do estado, sendo este um fator limitante do presente estudo, podendo haver subnotificação. No entanto, este trabalho irá contribuir de forma significativa para o planejamento e implementação de medidas de controle e de vigilância epidemiológicas mais efetivas contra a COVID-19 no Estado do Pará.

Logo, para os trabalhos futuros, sugere-se que busquem mais dados sócio demográficos. No presente estudo, utilizouse densidade populacional, IDH e tratamento de esgoto, contudo existem outros dados que podem ser analisados e correlacionados com a COVID-19, como porcentagem de água tratada e porcentagem de população urbana e rural.

\section{Agradecimentos}

Nossos agradecimentos ao Grupo de Estudos e Pesquisa em Saúde e Educação na Amazônia - GEPSEA, ao qual a equipe deste artigo pertence, à Universidade Federal do Pará, Campus Universitário de Altamira e à Secretaria Estadual de Saúde Pública (SESPA).

\section{Referências}

Andrade, S. S. (2019) Políticas Públicas Na Amazônia Marajoara: Os Índices De Desenvolvimento Socioeconômico Na Região. Nova Revista Amazônica. 7(1):159-179. 10.18542/nra.v7i1.6981

Barata, R. C. B. (1997) O desafio das doenças emergentes e a revalorização da epidemiologia descritiva. Rev. Saúde Pública. 31(5):531-537.

Beaglehole, R., \& Bonita, R. (1997) Public health at the crossroad: Cambridge University Press.

Benvenuto, D., Giovanetti, M., Ciccozzi, A.; Spoto, S., Angeletti, S., \& Ciccozzi, M. (2020) The 2019-new coronavírus epidemic: evidence for virus evolution. J Med Virol. 92(9):455-460.

Cai, H. (2020) Sex difference and smoking predisposition in patients with COVID-19. Lancet Respir Med. 8:e20.

Cascella, M., Rajnik, M., Cuomo, A., Dulebohn, S., \& Di Napoli, R. F., (2020) Evaluation and Treatment Coronavirus (COVID-19): StatPearls Publishing.

Chan, J. F. W., Yuan, S., Kok, K. H., To, K. K. W., Chu, H., Yang, J., Xing, F., Liu, J., Yip, C. C. Y., Poon, R. S. W., Tsoi, H. W., Lo, S. K. F., Chan, K. H., Poon, V. K. M., Chan, W. M., Ip, J. D., Cai, J. P., Cheng, V. C. C., Chen, H., Kim-Ming Hui, C. K. M., \&Yuen, K. W. (2020) A familial cluster of pneumonia associated with the 2019 novel coronavirus indicating person-to-person transmission: a study of a family cluster. Lancet. 23(1):395-400.

Chen, N., Zhou, M., Xuan Dong, X., Qu, J., Gong, F., Han, Y., Qiu, Y., Wang, J., Liu, Y., Wei, Y., Xia, J., Yu, T., Zhang, X., \& Zhang, L. (2020) Epidemiological and clinical characteristics of 99 cases of 2019 novel coronavirus pneumonia in Wuhan, China: a descriptive study. Lancet. 395:507-13.

Dias, V. M. C. H., Cunha, C. A., Vidal, C. F. L., Corradi, M. F. D. B., Michelin, L., Muglia, V., Rocha, J. L. L., Costa, S. F., Oliveira, P. R. D., Carrilho, C. M., Chebabo, A., Nunes, R. R., Diego, L. A. S., Santos, A. S., Carneiro, M., Souza Junior, A. S., Escuissato, D., Araújo Neto, C., Waib, L. F., Martins, R., Maurici, R., Chatkin, .M., Brandão, D., Lobo, S. M. A., Oliveira, M. C., Alves, J. S., Nascimento, M. M., \& Moura-Neto, J. A. (2020) Orientações sobre Diagnóstico, Tratamento e Isolamento de Pacientes com COVID-19. J. Infect. Control. 9(2).

Doremalen, N. V., Morris, D. H., Holbrock, M. G., Gamble, A., Williamnson, B. N., Tamin, A., Harcourt, J. L., Thornbourg, N. J., Gerber, S. I., LloydSmith, J. O., Wit, E., \& Munster, V. J. (2020) Aerosol and Surface Stability of SARS-CoV-2 as Compared with SARS-CoV-1. N Engl J Med. 382:15641567.

Fundo das Nações Unidas para a Infância - UNICEF. (2011) Joint Monitoring Program: Progress on sanitation and drinking-water.

Instituto Brasileiro de Geografia e Estatística - IBGE. Cidades e Estados 2010. < https://www.ibge.gov.br/cidades-e-estados >.

Instituto Trata Brasil - ITB (2020) Painel Saneamento Brasil 2020. Ranking do Saneamento. Instituto TrataBrasil.

Jaillon, S., Berthenet, K., \& Garlanda, C. (2019) Sexual dimorphism in innate immunity. Clin Rev Allergy Immunol 56(3):308-321.

Kerr-Pontes L. R. S., Barreto, M. L., Evangelista, C. M. N., Rodrigues, L. C., Heukeuback, J., \& Fedmeier, H. (2006) Socioeconomic, environmental, and behavioural risk factors for leprosy in North-east Brazil: Results of a case-control study. International Journal of Epidemiology. 35(4):994-1000.

Lerderberg, J. (1997) Infectious disease as an evolutionary paradigm. Emerg Infect Dis. 3(4):417-23. 10.3201/eid0304.970402.

Lima, D. L. F., Dias, A. A., Rabelo, R. S., Cruz, I. D., Costa, S. C., Nigri, F. M. R., \& Neri, J. R. (2020) COVID-19 no estado do Ceará, Brasil: comportamentos e crenças na chegada da pandemia. Ciência \& Saúde Coletiva. 25 (5): 1575 - 1586. 10.1590/1413-81232020255.07192020

Lima-Costa, M. F., \& Barreto, S. M. (2003) Tipos de estudos epidemiológicos: conceitos básicos e aplicações na área do envelhecimento. Epidemiologia e Serviços de Saúde. 12(4): 189. 
Lopes, L. F. D., Faria, R. M., Lima, M. P., Kirchhof, R. S., Almeida, D. M., \& Moura, G. L. (2020) Descrição do Perfil Epidemiológico da COVID-19 na Região Sul do Brasil. Revista Brasileira de Geografia Médica e da Saúde Hygeia. 16:188-198.

Luna, E. J. A., \& Silva Jr, J. B. (2013) Doenças transmissíveis, endemias, epidemias e pandemias. A saúde no Brasil em 2030 - prospecção estratégica do sistema de saúde brasileiro: população e perfil sanitário [online]: Fiocruz / Ipea / Ministério da Saúde. 2: 123-176.

Maciel, J. A. C., Castro-Silva, I. I., \& Farias, M. R. (2020) Análise inicial da correlação espacial entre a incidência de COVID-19 e o desenvolvimento humano nos municípios do estado do Ceará no Brasil. Rev. bras. epidemiol. 23. 10.1590/1980-549720200057.

Mascarenhas, M. D. M., Batista, F. M. A., Rodrigues, M. T. P., Barbosa, O. A. A., \& Barros, V. C. (2020) Ocorrência simultânea de COVID-19 e dengue: o que os dados revelam? Cad. Saúde Pública 36(6):17. 10.1590/0102-311X00126520.

Ministério da Saúde - MS. (2009) Influenza. Guia de Vigilância Epidemiológica: Ministério da Saúde.

Ministério da Saúde - MS. (2020) Doença pelo Coronavírus COVID-19. Boletim Epidemiológico Especial 17: Ministério da Saúde.

Monteiro Neto, A., Brandão, C. A., Macedo, D. S. F. C., Camargo, k., Porto, L. R., Colombo, L. A., Silva, R. O., \& Carmo, R. L. (2017) Desenvolvimento Regional No Brasil: Políticas, Estratégias E Perspectivas: Instituto de Pesquisas Econômicas Aplicadas.

Muralidar, S., Ambi, S. V., Sekaran, S., \& Krisnan, U. M. (2017) The emergence of COVID-19 as a global pandemic: Understanding the epidemiology, immune response and potential therapeutic targets of SARS-CoV-2. Biochimic. 179(2020): 85-100. 10.1016/j.biochi.2020.09.018.

Silva, A. W. C., Cunha, A. A., Alves, G. C., Corona, R. A., Dias, C. A. G. M., Nassiri, R., Vedovelli, S., Vilhena, T. R. F., Favacho, V. B. C., Sousa, J. F., Araújo, M. H. M., Oliveira, E., Densdasck, C. V., \& Fecury, A. A. (2020). Perfil epidemiológico e determinante social do COVID-19 em Macapá, Amapá, Amazônia, Brasil. Revista Científica Multidisciplinar Núcleo do Conhecimento. 4(04): 05-27.

Nassiri, R. (2020) Perspective on Wuhan Viral Pneumonia. Advances in Public Health, Community and Tropical Medicine. 2.

Organização Mundial de Saúde - OMS/WHO. (2020) Report of the WHO-China Joint Mission on Coronavirus Disease 2019 (COVID-19): World Health Organization.

Organização Mundial de Saúde - OMS/WHO. (2005) International Health Regulations: World Health Organization.

Organização Mundial de Saúde - OMS/WHO (2020). Coronavirus Disease (COVID-19). Dashboard WHO: World Health Organization.

Pachiega, J., Afonso, A. J. S., Sinhorin, G. T., Alencar, B. T., Araújo, M. S. M., Longhi, F. G., Zanetti, A. S., \& Espinosa, O. A. (2020) Chronic heart diseases as the most prevalent comorbidities among deaths by COVID-19 in Brazil. Rev Inst Med Trop São Paulo. 2020;62:e45 P.

Pereira, A. S., Shitsuka, D. M., Parreira, F. J., \& Shitsuka, R. (2018). Metologia da pesquisa científica. [Ed. UAB/NTE/UFSM. https://repositorio.ufsm.br/bitstream/handle/1/15824/Lic_Computacao_Metodologia-Pesquisa-Cientifica.pdf?sequence=1.

Secretaria de Saúde Pública do Estado do Pará - SESPA. (2020). Monitoramento COVID-19. Sistema de Vigilância da Secretaria de Saúde Pública do estado do Pará.

Silva, D. F., \& Oliveira, M. L. (2020) Epidemiologia da COVID-19: Comparação entre boletins epidemiológicos. Com. Ciências Saúde. 31 Suppl 1:61-74

Sousa, R. C., Ferreira, L. F. B., Ribeiro, M. E. S., Ferreira, A. C. D., Ferreira, D. V. G., Assis Neto, C. M., Cavalcante, R. L., Souza, G. F., Pinheiro, M. C. N., Sousa, A. A., \& Silva Júnior, A. F. (2020) Spatial distribution and incidence of covid-19 cases in indigenous populations in the xingu River Region, Pará, Brazil. International Journal of Development Research. 10:8.

Souza, D. O (2020) A pandemia de COVID-19 para além das Ciências da Saúde: reflexões sobre sua determinação social. CSC. 25(supl.1).

Souza, L. P. (2020) O Uso da Telessaúde durante a Pandemia da COVID-19: Scoping Review e uma Reflexão sobre o Atual Cenário Brasileiro. COVID-19 no Brasil: os múltiplos olhares da ciência para compreensão e formas de enfrentamento. Atena Editora, 2020.

Waldman, E. A., \& Sato, A. P. S. (2016) Trajetória das doenças infecciosas no Brasil nos últimos 50 anos: um contínuo desafio. Rev Saúde Pública. $50: 68$.

Wang, D., Hu, B., Hu, C., Zhu, F., Liu, X., Zhang, J., Wang B., Hui X., Cheng, Z., Xiong, Y., Zhao, Y., Li, Y., Wang, X., \& Peng, Z. (2020) Clinical characteristics of 138 hospitalized patients with 2019 novel coronavirus-infected pneumonia in Wuhan, China. JAMA. 323(11): 1061.

Zhu, N., Zhang, D., Wang, W., Li, X., Yang, B., Song, J., Zhao, X., Huang, B., Shi, W., Lu, R., Niu, P., \& Zhan, F. (2020) China Novel Coronavirus Investigating and Research Team. A Novel Coronavirus from Patients with Pneumonia in China, 2019. N Engl J Med. 382(8): 727-33. 